\title{
Application of contactless ultrasound toward automated inspection of concrete structures
}

\author{
Suyun Ham ${ }^{\mathrm{a}}$, and John S. Popovics ${ }^{\mathrm{b}^{*}}$
}

a Graduate Research Assistant, Department of Civil and Environmental Engineering, The University of Illinois at Urbana-Champaign, 205 N. Mathews Ave, MC-250, Urbana, Illinois 61801, USA

b Associate Professor, Department of Civil and Environmental Engineering, The University of Illinois at Urbana-Champaign, 205 N. Mathews Ave, MC-250, Urbana, Illinois 61801, USA

* Corresponding author (johnpop@illinois.edu ; tel. (217) 244 0843)

Keywords: air-coupled, infrastructure, MEMs, microcracking, nondestructive, rail ties, sensor, surface waves.

\section{ABSTRACT}

In this paper we demonstrate the potential of contactless ultrasonic sensing for rapid and automated characterization of concrete structures. Practical generation and detection of ultrasonic surface waves is made possible using air-coupled sensors. The air-coupled sensors obviate the need for the transmitter and receiver to be in physical contact with concrete, offering the potential to overcome limitations associated with such measurements. Although air-coupled ultrasonic techniques have been studied for some time, this paper addresses key technical barriers to the implementation of contactless ultrasonic testing to concrete infrastructure elements that have recently been overcome. The objective is to demonstrate enhanced performance when miniature, micro-machined sensors (MEMS) and high voltage solid-state capacitance transmitters are used in a scanning configuration, suitable for automation. The employed sensors, system and testing configuration, with respect to sensor height and incident angle, are described. An optimal test configuration is defined and incorporated into a controlled scanning system. Tests with the optimized configuration were carried out on reinforced concrete elements: a pre-stressed concrete rail tie that contains rail seat damage and concrete blocks with varying levels of simulated micro-cracking damage. In both cases, obtained surface wave velocity and attenuation signal characteristics show sensitivity to concrete material damage. 


\section{Introduction and motivation}

Rapid in-place structural damage characterization of large or extensive concrete infrastructure elements has become an issue of great importance for those responsible for their management [1-3]. Automated inspection procedures are desirable, especially considering the massive and often inaccessible nature of concrete infrastructure elements, as illustrated in Figure 1. For example, concrete is widely used in nuclear power generation structures, which under normal operating conditions may be exposed to aggressive environmental exposure (moisture and contaminating ions), high temperature hot spots at piping penetrations, turbine foundations and shield walls within the primary containment structure, and sustained radiation exposure [4]. Another example of important concrete infrastructure elements is rail ties for high-speed rail systems. High-speed rail systems demand increased track structure stability, so the physical condition of the rail track structure must be regularly monitored in order to ensure its safe and operating efficiently [5]. Though significant progress in health monitoring and non-destructive testing of large concrete infrastructure systems has been made over the years [6], reliable methods for rapid and effective material characterization of concrete in the particular systems described have not yet been realized.

Most conventional concrete non-destructive test (NDT) methods currently employed, such as visual inspection, sounding (e.g., chain drag), pulsed microwave/radar techniques (GPR) and infrared thermography, cannot reliably detect and characterize internal distributed damage in concrete [6]. Mechanical wave methods, such as ultrasonic wave pulse propagation, do show sensitivity to distributed damage, and can be applied to large structures in the field. However, conventional ultrasonic methods utilize sensors that require physical contact and proper coupling with the concrete structure. This sensor coupling process is both time and labor intensive, and thus prohibitively slow to carry out. When the concrete surface is rough, surface preparation (e.g., grinding) is needed prior to testing, and in extreme cases tests simply cannot be applied. Furthermore, the sensor coupling conditions may affect received signals and disrupt measurements. Thus, conventional ultrasonic tests on concrete show limitations owing to the need for physical coupling.

The deployment of contactless (air-coupled) ultrasonic sensors offers potential to overcome some of these limitations and to provide a pathway for automated rapid inspection of concrete infrastructure elements. Contactless ultrasonic tests have been successfully applied to inspect a wide range of materials, where ultrasonic wave speed or signal energy attenuation are normally measured and material properties inferred [7]. The first applications of contactless ultrasound to concrete and other cement-based materials involved the use of compressional waves (p-waves). Air-coupled sensors were positioned on opposing sides of a sample in a through-thickness configuration, and the airborne 
ultrasound p-wave pulses were projected normal to the surface of the samples [8-11]. Although generally good results were obtained by these investigators, the method is limited by the fact that access to opposing sides is required and, further, only relatively thin samples (less than or equal to 10 $\mathrm{cm}$ ) can be tested because of the low signal amplitudes that were received. Furthermore, the signal-tonoise ratio (SNR) of the data is low, so additional signal processing (e.g. signal averaging and wavelet analysis) is usually required. The signals also suffer from internal wave scattering in concrete when higher frequencies (> $200 \mathrm{kHz})$ are employed.

The application of ultrasonic surface waves to concrete followed, allowing tests on samples with only one-sided access. In these tests, the air-coupled transducers are located on the same side of the sample, where the airborne ultrasonic energy is usually projected at the surface at some non-normal "critical" angle to promote surface wave generation. Air-coupled, contactless surface wave tests have been used in laboratory test studies to monitor porosity or cracking in small cement paste [12], mortar [13], and concrete [14-16] samples, and to characterize aggregate distribution in asphalt concrete [17]. In some cases, sophisticated signal analyses schemes were employed, such as dispersion analysis with slantstack transforms [14] and non-linear analysis [16], which suggest that high quality signal data can be obtained. Preliminary but limited application of air-coupled ultrasonic tests to a concrete structure in situ has been reported [15]. However, field application of air-coupled ultrasonic tests remains uncommon. Abraham et al. [14], Piwakowski [15] and Garnier [16] used the same commercial aircoupled ultrasonic testing hardware system, where low SNR signals and high sensitivity to the surface condition are reported for some cases.

This paper presents work conducted to overcome key technical barriers to automated contactless ultrasonic testing implementation in concrete infrastructure elements. In particular, we evaluate the utility surface-guided waves for this purpose. First, we introduce and optimize a contactless ultrasonic testing configuration that uses low cost and practical sensors so that multi-sensor arrays may be more readily deployed. The testing system produces high quality (high signal-to-noise ratio) signal data and enables reasonably large inspection lengths, e.g. greater than $0.5 \mathrm{~m}$, in conventional concrete without necessitating surface preparation. We deploy the contactless ultrasonic testing on a pre-stressed concrete rail tie that contains realistic damage conditions to demonstrate the utility of the ultrasonic tests on concrete infrastructure elements. We also study the utility of the method for characterizing distributed cracking damage in concrete. In both cases, the ultrasonic testing, signal processing and analyses schemes are designed to be appropriate for incorporation within an automated scanning system. The findings reveal potential for application air-coupled ultrasonic testing to concrete infrastructure elements in the field and demonstrate new testing capability for concrete structures, enabling exciting possibilities for improved automated or robotic inspection of the concrete 
infrastructure.
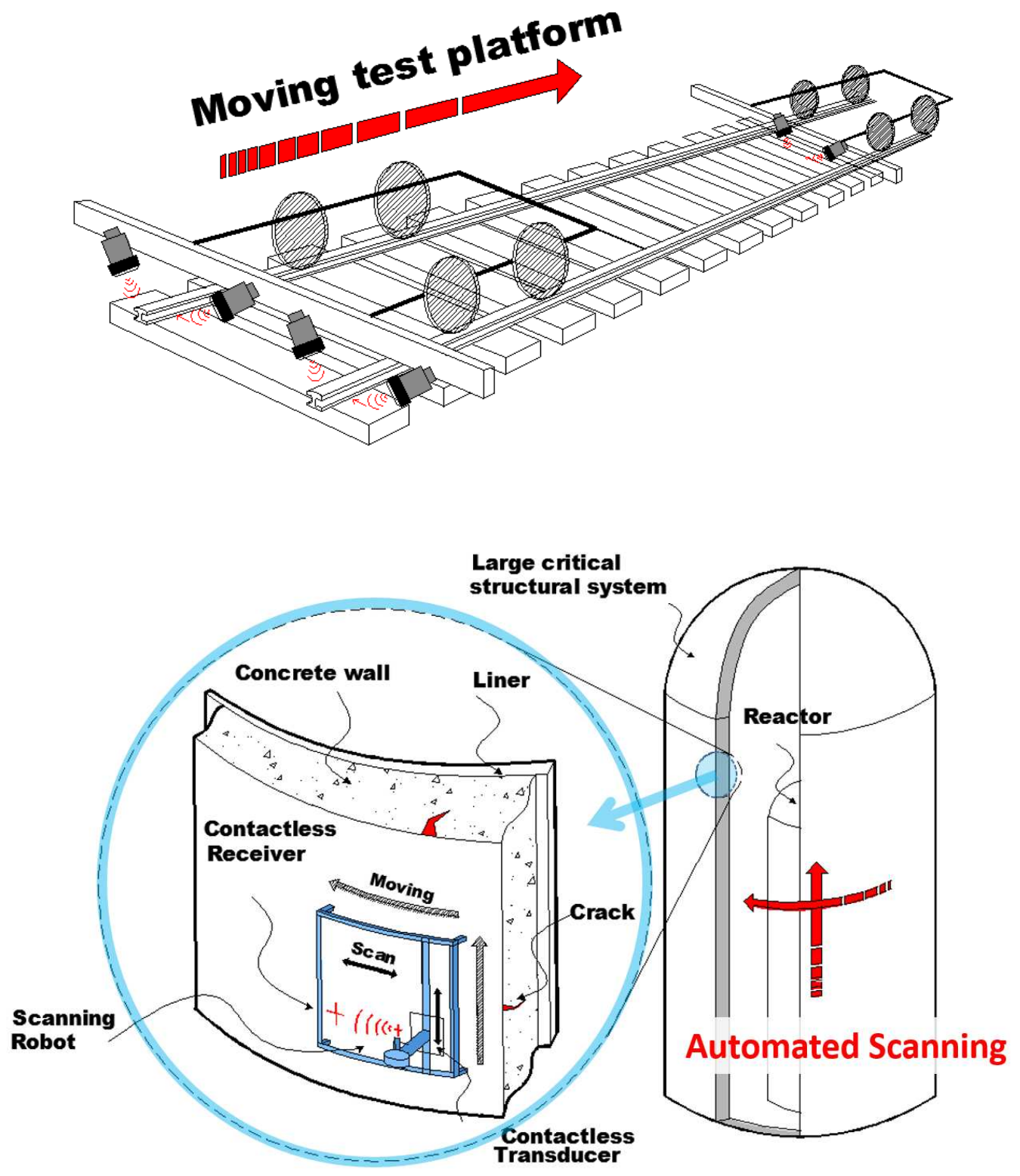

Figure 1. Deployment concepts for fast and accurate air-coupled ultrasound inspection of (top) concrete rail ties from a moving platform and (bottom) large structural systems such as a nuclear power plant containment dome using a scanning robot. 


\section{Background information}

\subsection{Surface-guided waves}

Surface guided waves, known as "Rayleigh" waves for the case of large material thicknesses with respect to the propagating wavelength, have unique advantages over longitudinal ( $\mathrm{p}$-waves) and shear (s-waves) body waves. Rayleigh waves can be sent and received when only one side of the concrete structure is accessible, and a certain degree of control of the penetration depth of the wave can be maintained through wave frequency [19]. Most of the Rayleigh wave energy is contained within a thickness zone of approximately one wavelength below the surface, and this size is often referred to as the wave "penetration depth" [20]. Given that the length and frequency of the wave are inversely related to each other through the wave phase velocity, the penetration depth of a $50 \mathrm{kHz}$ Rayleigh wave in concrete is approximately $5.5 \mathrm{~cm}$, assuming a typical Rayleigh wave phase velocity of 2750 $\mathrm{m} / \mathrm{s}$. Thus along a given surface wave path, a volumetric region defined by the surface region penetration depth along the path between the sender and receiver is defined. This characteristic allows us to assign surface wave signal characteristics to spatially discrete regions within a sample, and thus provides the potential to deploy image visualization schemes to re-construct maps of damage in the structure using surface wave data. Finally, the fact that the wave energy is maintained within a finite zone near the surface dictates that surface waves suffer lower amounts of geometrical energy loss (owing to beam spreading) and, thus, can propagate over larger distances for a given amount of energy loss than can body waves [20].

\subsection{Air-coupled ultrasonic tests}

When a propagating body wave (p-wave or s-wave) interacts with interface across which the mechanical properties of the material change, some of the incident wave energy will reflect back away from the interface, and the rest will refract forward through the interface. Also, the wave will undergo mode-conversion, meaning that p- and s-wave, and in some cases Rayleigh wave, components will be present in both reflected and refracted waves, regardless of the nature of the incident wave. The amplitude and type of reflected and refracted waves depend on the relative wave velocities of the two media and the angle of incidence of the wave through Snell's law. Beyond a certain "critical" angle of incidence, $\theta_{\mathrm{C}}$, no waves of a certain mode type are transmitted into the second medium. Assuming that a p-wave travelling in a fluid (e.g. air) is incident on the surface of a solid material (e.g. concrete), Snell's law defines the first critical angle of reflection, $\theta_{\mathrm{C} 1}$, to be

$$
\theta_{C 1}=\arcsin \left(\frac{C_{f}}{C_{P}}\right)
$$

where $\mathrm{C}_{\mathrm{f}}$ is the velocity of wave propagation in the fluid and $\mathrm{C}_{P}$ is the $\mathrm{p}$ - wave velocity in the solid. 
When the p-waves are incident at an angle beyond $\theta_{\mathrm{C} 1}$, no p-wave energy is refracted forward into the solid material. The second critical angle, analogously, defines the incident angle beyond which swaves (and also p-waves) are not refracted forward into the solid material. At incident angles close to the second critical angle, a pseudo-pressure wave builds and propagates along the surface. Rayleigh waves are generated when the wave in the fluid wave is incident at an angle slightly greater than the second critical angle. This angle, $\theta_{\mathrm{R}}$, is given by Snell's law

$$
\theta_{R}=\arcsin \left(C_{f} / C_{R}\right)
$$

where $C_{R}$ is Rayleigh wave velocity. The generated Rayleigh waves that propagate along the surface of the solid at the fluid interface leak energy in the form of p-waves back into the fluid at the same angle $\theta_{\mathrm{R}}[21]$.

\subsection{Air-coupled ultrasonic sensors}

Ultrasonic transducers that generate waves in air are usually based on one of two types of technology: resonant piezoelectric ceramic (PZT) solid elements or a capacitive membranes [22]. PZT elements are excited with a transient electrical pulse, upon which the PZT element vibrates at the natural resonant frequencies of the element. Capacitive membrane, or electrostatic, transducers contain a metal dielectric membrane that is positioned with its insulating side against the surface of a rigid conducting back plate [23]. PZT transducers operate at lower voltage, enabling simple electronic design, while capacitive membrane transducers require a high biasing voltage above $100 \mathrm{~V}$. Capacitive membrane transducers generally exhibit broader frequency response compared to piezoelectric transducers, which have resonant (high-Q) ceramic crystal that produce a relatively narrow frequency response, although modifications to the transducer design can be carried out in order to generate signals with a broader band of frequencies [24]. PZT transducers tend to be inefficient airborne wave energy generators because of the large acoustic impedance mismatch at the PZT-air boundary and the relatively low level of excitation power that can be sustained by the PZT material $[25,26]$.

Ultrasonic transducers that detect (or receive) propagating p-waves in air are normally either PZT or capacitive type transducers. Other sensing technologies, for example microphones of varying types, also can detect propagating waves in air. Microphones typically exhibit high sensitivities, about 2 $\mathrm{mV} / \mathrm{Pa}$ at $1 \mathrm{kHz}$. However the working frequency range depends on the type of microphone used; conventional microphones are limited to frequencies below $20 \mathrm{kHz}$, while electret condenser microphones (ECM) can sense, in some cases, up to $70 \mathrm{kHz}$. Recent developments in miniature, micro-machined sensors (MEMS) technology, provide new a sensing basis for airborne ultrasound. MEMS sensors operate as a capacitive transducer, using a thin silicon nitride diaphragm. The advanced developments behind MEMS offer reduced power consumption, improved performance in 
varied environmental conditions, reduced weight and smaller form factors, and are lower cost than their traditional sensing counterparts [27]. Many types of sensors, except for the MEMS type, have been used to detect airborne ultrasonic waves from concrete $\quad[14,28,29]$. However capacitive MEMS sensor overcome limitations in bandwidth and uniformity of response of traditional air-coupled acoustic sensing technology such as ECM and PZT sensors [30].

\subsection{Damage in concrete}

Microcracks in concrete can be caused by the alkali-silica reaction (ASR), thermal expansion, freezing and thawing action, other chemical reactions, and mechanical actions such as fatigue or overloading. ASR damage is a reaction which occurs over time in concrete between the highly alkaline cement paste and reactive non-crystalline (amorphous) silica, found in many common aggregates [31]. Freezing and thawing action occurs in concrete that comes into contact with moisture, rendering it susceptible to damage under repeated cycles of freezing and thawing (frost attack) [31]. If there is no space for this volume expansion in a porous, water-containing material like concrete, freezing may cause distress in the concrete. Chemical attack damage results, for example, from carbonation, and sulfate attack. Carbonation occurs when carbon dioxide from air reacts with the calcium hydroxide in concrete to form calcium carbonate. Carbonation of concrete is a slow, continuous process progressing from the outer surface inward, slowing with increasing diffusion depth. Sulfates in solution in contact with concrete can cause chemical changes to the cement, which can cause significant microstructural effects leading to the weakening of the cement binder (chemical

sulfate attack). In the case of concrete rail ties, the most pressing problem for heavy haul service has been reported to be rail seat deterioration (RSD) [32]. This problem is especially acute for the major railroads, with their higher traffic volumes and heavier axle loads, as RSD may eventually cause derailment accidents. RSD is caused by a complicated failure mechanism that involves multiple contributing factors, including lateral surface abrasions and internal hydraulic hydrostatic pressure impulses within the concrete pore fluid caused by passing train loads. These damage mechanisms eventually lead to a crushing type of concrete deterioration, resulting in unbound material and exposed aggregates at the concrete surface underneath the rail pad. As such, RSD is distinct from cracking forms of damage that result from convention deterioration mechanisms.

\section{Ultrasonic testing system}

In this paper, ultrasonic surface guided waves in concrete are emitted and detected using a fully contactless air-coupled system, which was designed and assembled by the first author. The fundamental concept and equipment configuration are shown in Figure 3. The technique is completely 
contactless as air-coupled sending and receiving transducers are employed. The equipment set comprises a computer, multi-channel data acquisition system, air-coupled capacitive transmitting transducer and air-coupled MEMS receiving transducer
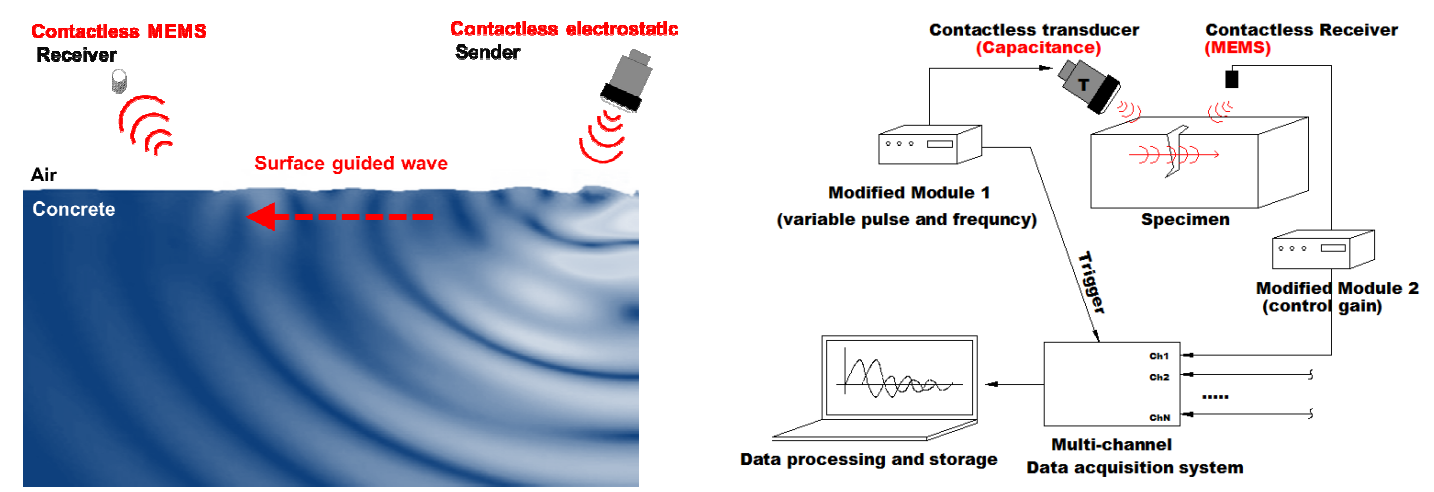

Figure 2. (left) illustrates concept of the fully contactless ultrasonic configuration and (right) shows testing setup.

\subsection{Modified air-coupled sending transducer}

The capacitive membrane transducer in our system is composed of a very thin Kapton-film membrane (or diaphragm), vacuum-coated with metal to form a negative electrode. The thin foil is movable, and transforms electrical energy into mechanical energy (air pressure) and, conversely, mechanical energy into electrical energy. The positive electrode is the coining aluminum back plate, which also provides the resonant structure for the membrane. A DC bias is applied externally by an electronic module that uses a high voltage transformer and a bipolar junction transistor (BJT). A steel spring transfers the voltage from the back plate and holds the membrane under constant tension. Then, an AC voltage of a given frequency is applied to the membrane, which forces the film to move at the same frequency, sending out acoustic waves. When acting as a detector, the arrival of an ultrasonic wave at the membrane varies the capacitance and, in the presence of an imposed bias field, generates a dynamically varying charge upon the electrodes. As described in section 2.3, capacitance transduction provides a far better match to air (compared with PZT sensors), as the air-pocket / membrane system has a much lower acoustic impedance [33]. Our capacitive transducer with a Kapton-film membrane is commercially available and is relatively inexpensive.

The electrical excitation system, the excitation module, that comes with the commercially available capacitive package plays an important role. The module generates a $50 \mathrm{kHz} 16$-cycle excitation signal. A "mid-frequency" band (20-100 kHz) of ultrasonic frequencies is desired for testing concrete. In this paper, two modified electrical excitation modules are introduced to control the pulse duration and frequency; these modules allows generation of variable pulse excitation that provides performance 
comparable to commercially available function generators. The first module, module A, comprises several ceramic resonators and a microchip. The module allows bandwidth and frequency control by a specific ceramic resonator. The ceramic resonator controls the central frequency of the capacitive transducer between 30 and $80 \mathrm{kHz}$. The microchip in the module controls pulse duration. The main microchip (generic microcontroller) for the pulse control is the ATMEL Tiny45, which is controlled with an open source code communicating with a computer through the AVR ISP connector. The 7805 IC regulator in the module maintains a stable and constant voltage level, reducing the electric noise in the built-in commercial module. Module A is simple and easy to construct, although the pulse duration must be at least 10 cycles long owing to limited sampling resolution of the microchip. Thus it is ideal for tone burst signals at a selected central frequency.

A second module, called module B, is also used. It generates arbitrary electrical excitation signals. A generic microcontroller, such as a single-board microcontroller or PIC (Peripheral interface controller), cannot create high frequency arbitrary waves having very low sampling rate, and as such are unsuitable for our purposes. Rather, field-programmable-gate-arrays (FPGA) are used to generate the arbitrary signal. FPGA operate using customizable logic gates instead of writing instruction sets to the board, like a microcontroller does. The Module B FPGA uses a Papilio One board with a Spartan 3E FPGA chip. Logic gates exhibit very fast response times, in the order of nanoseconds; this is sufficient to create arbitrary pulses up to a frequency of $200 \mathrm{kHz}$. Furthermore, it is easily programmed so any waveform can be produced. Programming is carried out using Xilinx's ISE Design Suite. Although the module is more complicated in its design, it is suitable for generating arbitrary waves and chirp signals.

\subsection{Modified air-coupled receiving transducer}

This effort uses a newly developed silicon-based miniature MEMS acoustic sensors to detect leaky ultrasonic surface waves in concrete. A variety of these sensors were obtained, configured, and evaluated by direct comparison to traditional microphones and capacitive sensors, using a consistent air-coupled ultrasonic source for tests on a concrete sample. The MEMS sensor is commercial product, but it does require power (1.5 3 DC volts) and ceramic capacitor connection. The MEMS sensor requires additional circuit design with a coupling $0.1 \mu \mathrm{F}$ capacitor at the output forming a high pass filter with input resistance. Our designed MEMS sensor has $1 \mathrm{~V} / \mathrm{Pa}$ sensitivity at $1 \mathrm{kHz}$ and a working frequency range of 1 to $90 \mathrm{kHz}$.; this represents higher sensitivity and a broader frequency band than other air-coupled sensors such ECM and dynamic microphones. CMOS (complementary metal-oxidesemiconductor) technology processes the output signals sensed from the MEMS membrane. Our MEMS is a monolithic CMOS MEMS sensor that combines MEMS with CMOS micro scale 
electronic circuitry. It is highly-integrated, having a small form factor $(2 \times 3 \mathrm{~mm})$, and represents a cost-effective solution as compared with conventional MEMS sensors. The reduced size provides improved spatial identification of the sensed point, so better quality inspection can be performed. In addition, the CMOS MEMS sensor (\$5 unit price) is far less expensive than a commercial condenser microphone ( $\$ 1600$ unit price), and a contact accelerometer, (\$400 unit price). The MEMS sensor does not require ancillary equipment, such as an amplifier. The small size and low unit cost enables deployment of many sensors in an array.

\subsection{Controlled scanning system}

A large amount of consistent and reliable (in terms of spatial position) fully contactless ultrasonic data must be collected in order to quantify spatial resolution of the system and to evaluate scanning and imaging suitability. For this study, we designed and constructed a testing frame robot, shown in Figure 3 that incorporates the air-coupled sending and receiving hardware. The auto scanning system (Figure 3 top left) provides precise, automated sensor positioning in the space above the concrete surface, achieving controlled sensor angle and height throughout the scanning process. This frame is part of an automated testing system that includes all necessary power, control, signal excitation, data acquisition and data storage capabilities. The auto test frame is designed to work with the proposed test samples and, furthermore, is adjustable and configurable to scan large-scale concrete samples or structures. The testing system was assembled in our laboratory using commercially available components and materials at reasonable cost.

A small 3-D printed frame (Figure 4, top right) is used to hold sending and receiving transducers. This small frame has light weight and allows accurate transducer positioning, incident angle and height. Once sufficient data sets are obtained, imaging protocols, for example signal parameter mapping, spectral signal stacking, and tomography, are then employed in order to build up amplitude attenuation images of the test samples. 

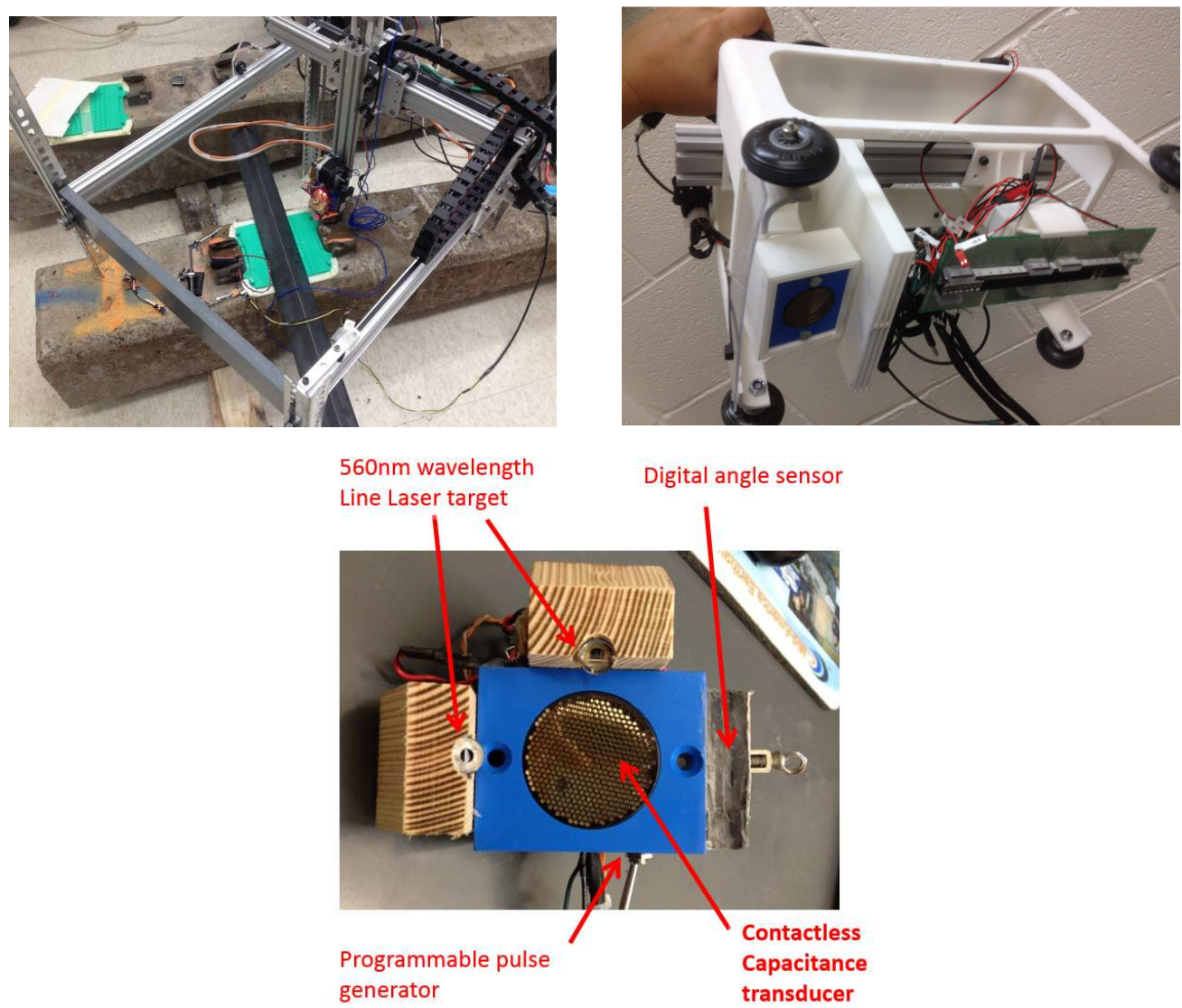

Figure 3. Photo of developed air-coupled scanning system: (top left) application of scanning system to a concrete element, (top right) $3 D$ printed sensor scanner, and (bottom) detail of transmitting transducer

\section{Results and Discussion}

The fully contactless ultrasonic testing configuration employed in this study is shown in Figure 5. "Height 1" indicates the distance between the concrete surface and the air-coupled sending transducer, and "Height 2" the distance between the surface and the air-coupled ultrasonic receiving transducer. The angle of incidence for the transmitter is indicated by "Angle." All test configuration parameters must be carefully controlled in order to optimize signal generation and detection. Several evaluation performance metrics with regard to signal generation are considered, including amplitude and bandwidth control, generated signal-to-noise ratio (SNR) and ease of incorporation into an automated scanning system. 


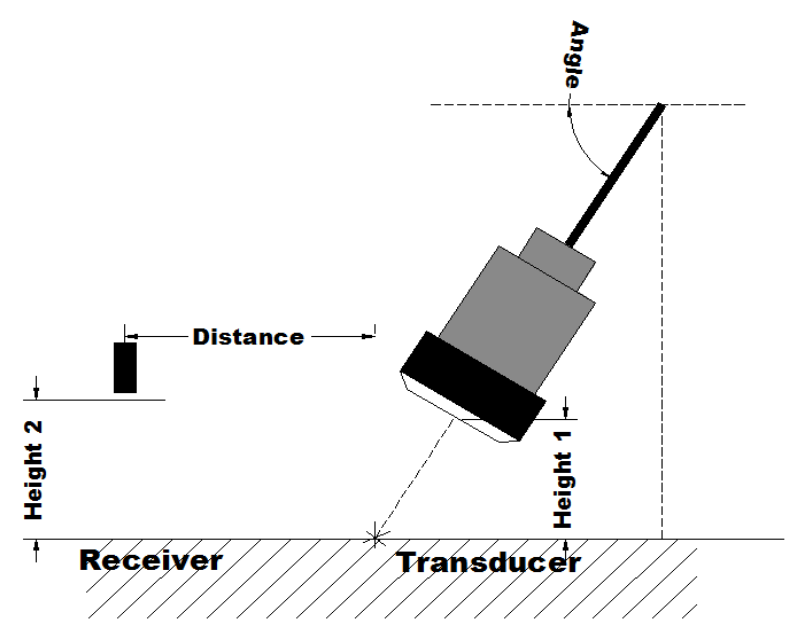

Figure 4. Illustration of testing configuration parameters for contactless set-up

\subsection{Optimizing sensor height and angle}

The air-coupled capacitive transmitter excites a $50 \mathrm{kHz}$ tone burst 16-cycle pulse using module A. Figure 5 (left) shows the effect of transmitting transducer height. Both the MEMS and contact sensors are used to detect the signal at a distance of $180 \mathrm{~mm}$, with Height 1 varying from $2 \mathrm{~mm}$ to $70 \mathrm{~mm}$ in increments of $2.5 \mathrm{~mm}$. These results indicate the highest surface air pressure amplitude at $52 \mathrm{~mm}$ of Height 1, as shown in Figure 5 (left). This coincides with the expected near-field distance for transducers. The near-field distance $(\mathrm{N})$ is approximated [34] by

$$
\mathrm{N}=\frac{D^{2} F}{4 V}
$$

where $D=$ diameter of the transducer, $F=$ frequncy of the transducer, and $\mathrm{V}=$ the velocity of sound in the material. With a $0.38 \mathrm{~m}$ diameter transducer at $50 \mathrm{kHz}$, Equation 1 gives a near-field distance approximation of $52.6 \mathrm{~mm}$.

In another test, the optimal angle of incidence for air-coupled sending transducer is studied. The sending transducer generates $50 \mathrm{kHz} 16$-cycle tone burst pulse using module $\mathrm{A}$. The incident angle is changed in $2.5^{\circ}$ increments from 72.5 to $90^{\circ}$, with a $180 \mathrm{~mm}$ distance and $52 \mathrm{~mm}$ for Height 1 . As shown in Figure 5 (right), the results indicate the highest surface wave amplitude for an angle of $82.4^{\circ}$. This experimental result is close to the theoretical critical angle of $81.0^{\circ}$, as described in section 2.2 . 


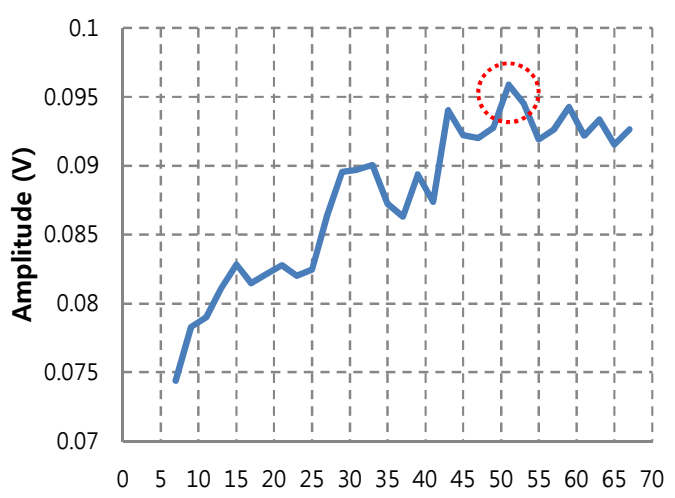

Height 1 (mm)

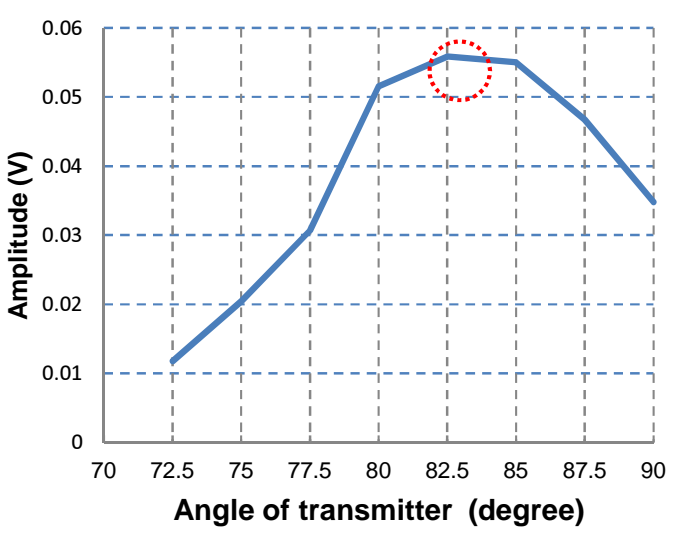

Figure 5. Effect of testing configuration parameters for sending transducer on amplitude of received signal with $5 \mathrm{~mm}$ for Height 2: (left) results indicate highest received amplitude at $50 \mathrm{~mm}$ sending height; (right) results indicate highest received amplitude for 82 degree sending angle.

\subsection{Comparison with contact sensors}

The performance of a variety of ultrasonic receivers, including an air coupled MEMS sensor, a electret condenser microphone (ECM), a dynamic microphone and a contact accelerometer, are studied using a consistent air-coupled ultrasonic sending configuration over a concrete sample. An AC capacitive transducer, excited with a $50 \mathrm{kHz}, 16$-cycle tone burst signal with module $\mathrm{A}$, was positioned at an $82.5^{\circ}$ angle of incidence and $52 \mathrm{~mm}$ height from the surface. The offset distance between transmitter and receivers is $280 \mathrm{~mm}$. The results are shown in Figure 6. The largest signal amplitude is provided by the contact accelerometer. The signal amplitude of the MEMS receiving transducer is approximately $1 / 6$ that of the contact sensor, and the condenser microphone shows much smaller signal amplitude, approximately 51 times lower than that obtained by the contact sensor. The dynamic microphone did not detect the surface wave signal; this was expected as the frequency range of those sensors extends only up to $20 \mathrm{kHz}$. The surface wave velocity in the concrete was $2150 \mathrm{~m} / \mathrm{s}$, and the acoustic wave velocity in the air, measured from arrival of direct acoustic wave, was $343 \mathrm{~m} / \mathrm{s}$. The MEMS sensor shows an excellent signal-to-noise ratio (SNR) at $34.4 \mathrm{~dB}$, while the condenser microphone shows $2.02 \mathrm{~dB}$ at $50 \mathrm{kHz}$. The contact accelerometer exhibits highest SNR at $61.2 \mathrm{~dB}$ at $50 \mathrm{kHz}$. The SNR is calculated as the ratio of variance of total signal to variance of noise in the signal. 


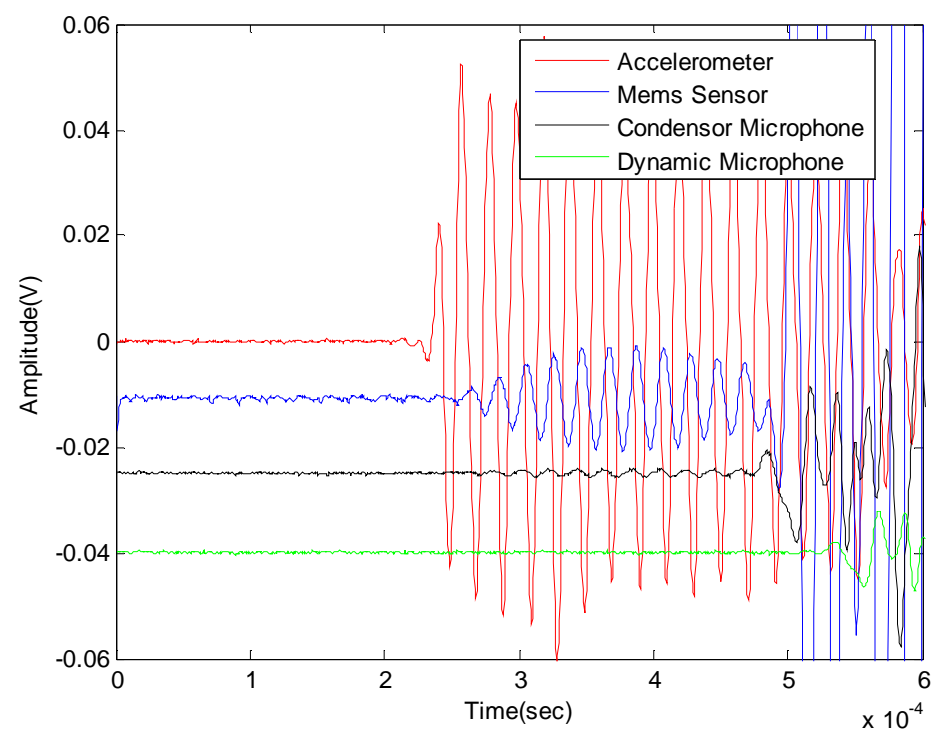

Figure 6. Comparison of received signals from different receiver types for a consistent source

An important ultrasonic testing performance issue is the consistency of the measured surface wave signal. Figure 7 (left) shows signals generated with the air coupled capacitive transducer under 50 $\mathrm{kHz}, 10$-cycle toneburst excitation with module $\mathrm{A}$ and collected by a single contact accelerometer. The source distance is $280 \mathrm{~mm}$. While maintaining consistent air-coupled surface wave generation, the accelerometer was attached then detached three times, successively, at the same position on a concrete sample. The results indicate the contact sensor does not provide consistent signal amplitude, as the signal amplitude varies significantly owing only to the coupling condition of the contact sensor. Figure 7 (right) shows signals collected from two different contactless MEMS receiving transducers over the same position on a concrete sample. The MEMS sensors show much more consistent surface wave signals, in contrast to the other sensors, which show higher variation. This suggests a notable benefit for contactless sensors within sensing arrays in a scanning system, where wave amplitude data are needed for example in signal attenuation measurements. 

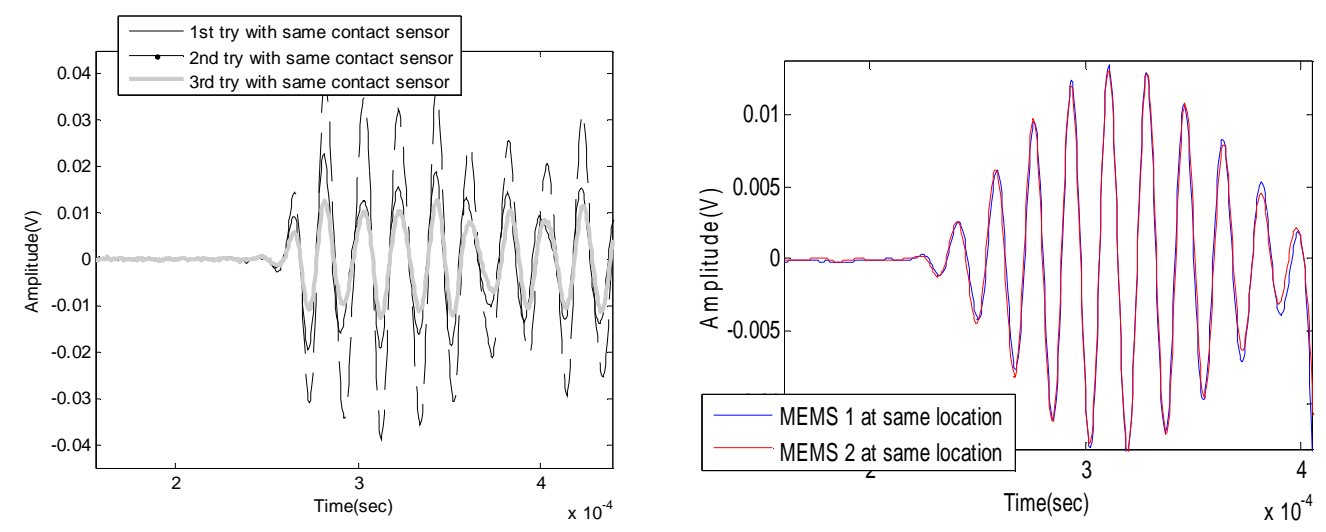

Figure 7. Illustration of sensor coupling consistency with regard to signal amplitude: (left) signals collected by a single contact accelerometer attached and detached three times at same position on a concrete sample using a consistent wave source, and (right) signals from two different contactless MEMS receiving transducers at the same position on a concrete sample

Another important testing characteristic is the application to rough material surfaces. The condition of concrete surfaces in the field can be variable, and ultrasonic systems should obtain viable signals regardless of surface condition. The surface roughness condition (surface spatial characteristics) can affect the ability to generate ultrasound in the material through two different phenomena: scattering of ultrasound and physical coupling of the active surface of the sending sensor to the material. Surface mounted sensors are affected principally by the latter effect, whereas air-coupled sensors are affected principally by the former. The air-coupled capacitive transducer, excited with $50 \mathrm{kHz}, 16$-cycle toneburst excitation with module A, was applied to concrete surfaces with three different roughness conditions that reasonably span the expected range for concrete structures. The source distance is $380 \mathrm{~mm}$. The surface roughness is quantified by estimated average vertical spatial variation across the tested area, ranging from $0 \mathrm{~mm}$ to $7 \mathrm{~mm}$. As shown in Figure 8, our ultrasonic system provides clear viable signals for all the surface conditions, although the signal amplitude decreases as the surface roughness increases. The observed amplitude variation is most likely caused by incident ultrasound scattering from the surface, but this effect is moderate because the ultrasonic wavelength is about one order of magnitude larger than the characteristic roughness sizes. Because this air-coupled system is not meaningfully affected by the physical sensor-surface coupling problem, it may be applied effectively regardless of surface roughness condition. 

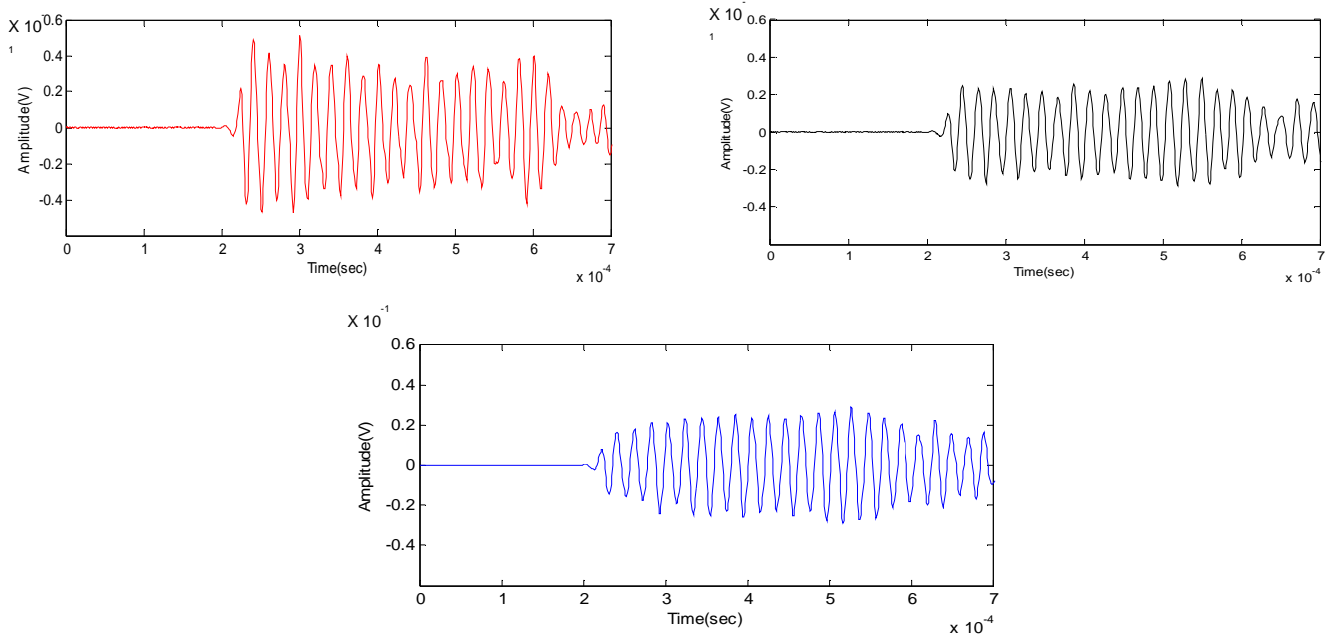

Figure 8. Air-coupled surface wave signals obtained from different surface roughness conditions in concrete: (top left) smooth $(0 \mathrm{~mm})$, (top right) medium level (2 mm average), and (bottom) extremly rough (5 $\mathrm{mm}$ average)

\subsection{Detecting damage in concrete rail ties}

An air-coupled ultrasonic testing configuration was used to inspect the two different concrete rail tie conditions, with and without RSD damage. The concrete samples are former in-service rail ties that were later extracted and evaluated by an expert as to the occurrence of RSD. RSD damage in ties is difficult to assess because the rail seat area is covered by a rail and an underlying pad. The ultrasonic transducers can access the ties only at the boundaries of the rail seats, as shown in Figure 9. Even with these limited accessibility issues, the fully air-coupled scanning ultrasonic system is able to collect data across the RSD area of the concrete tie. The air-coupled capacitive transducer was excited with a $60 \mathrm{kHz}, 5$-cycle excitation using module B. 

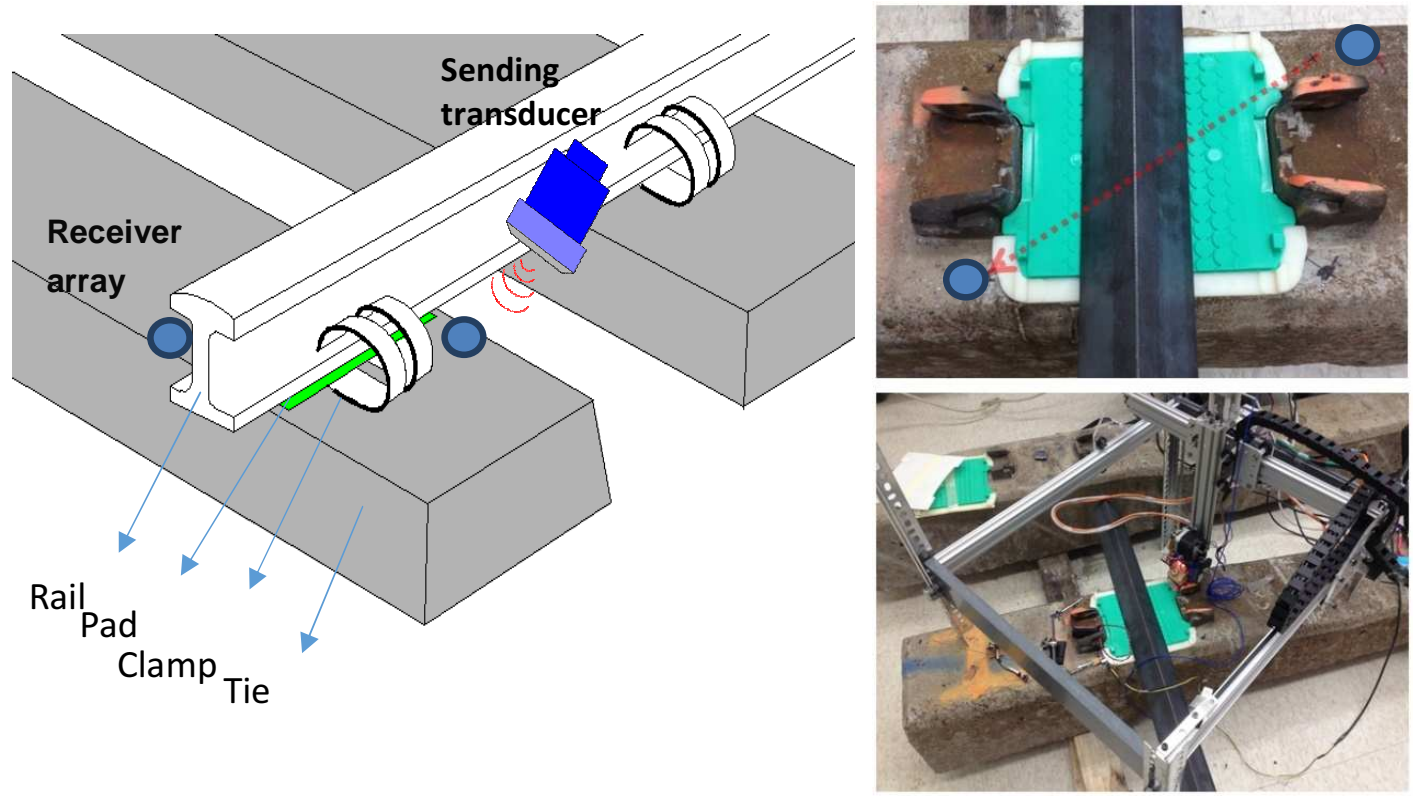

Figure 9 Rail seat testing configuration

Test results, shown in Figure 10, demonstrate that full-scale concrete tie samples with varying levels of RSD deterioration are distinguished. Ten signals are time averaged to produce one averaged time signal. The surface wave arrival times of both RSD damaged and undamaged samples are similar. However, the amplitude of fully air coupled UT signals provides clear distinction between no damage and RSD damage, where samples with RSD damage exhibit lower amplitude. The results show promise for the approach, but we are currently studying the basis for this observed behavior with ongoing research in an effort to develop an approach to characterize the extent of RSD from in-place ultrasonic measurements. 


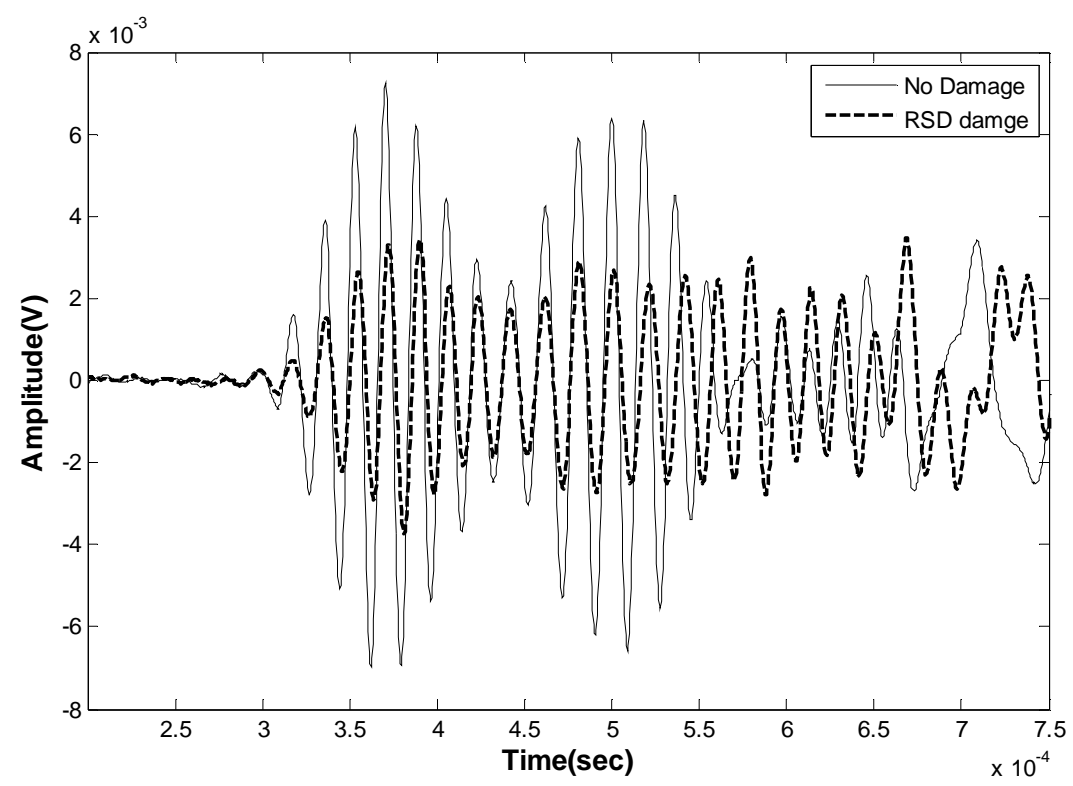

Figure 10. Comparison of received amplitude from different RSD damage conditions on the tie surface under the rail seat.

\subsection{Detecting distributed scatterers within concrete}

Distributed internal polymer fillers have been used by researchers to mimic cracking damage in concrete, where the fibers simulate internal reflectors for ultrasonic multiple scattering studies [35]. The filler (scatterer) volume can be apportioned in a controlled manner. Three different concrete blocks with varying mixture constituents, but having the same age, were used. The concrete contains varying amounts of discrete plastic polymer fibers that represent micro cracks within the concrete. Fully contactless and semi-contactless (with a contact accelerometer as reference) systems were used. Tests were carried out on three large concrete beam samples ( $300 \times 300 \times 600 \mathrm{~mm})$ having different mixture constituents. All concrete samples had identical age and curing conditions. They also had identical constituents except for randomly distributed polymer fiber content (soft scatterers): $0,0.3$ and $0.6 \%$ by volume. The compliant polymer fibers were used to simulate uniformly distributed cracking damage (air-filled micro-cracks and voids) in concrete in a controlled manner. $50 \mathrm{kHz}$, fully contactless ultrasonic surface waves were sent and detected along the top surface of each beam for three different path lengths, up to a maximum length of $400 \mathrm{~mm}$. Figure 11 shows stacked ultrasonic time signals (B-scan images) collected from each concrete sample across a 50mm sending distance using the optimal sending configuration with module B and sensed by a contactless MEMS sensor. The B-scan images show a clearly defined wave front that indicates the arrival of the surface wave. All arrival times are determined automatically using the Akaike information criterion (AIC) routine in 
the MATLAB platform. Tests revealed that the three beams had similar average surface wave velocities about $2160 \mathrm{~m} / \mathrm{s}$. Surface wave velocities obtained from spatially averaged tests, conducted separately with varying source distances, also exhibit velocities around $2160 \mathrm{~m} / \mathrm{s}$. Although reliable and high quality surface wave velocities were obtained, it was still difficult to distinguish the three different cracking contents based on each arrival time. The characterization of simulated microcracked concrete requires more sensitive measurements and precise analysis than velocity analysis alone provides.

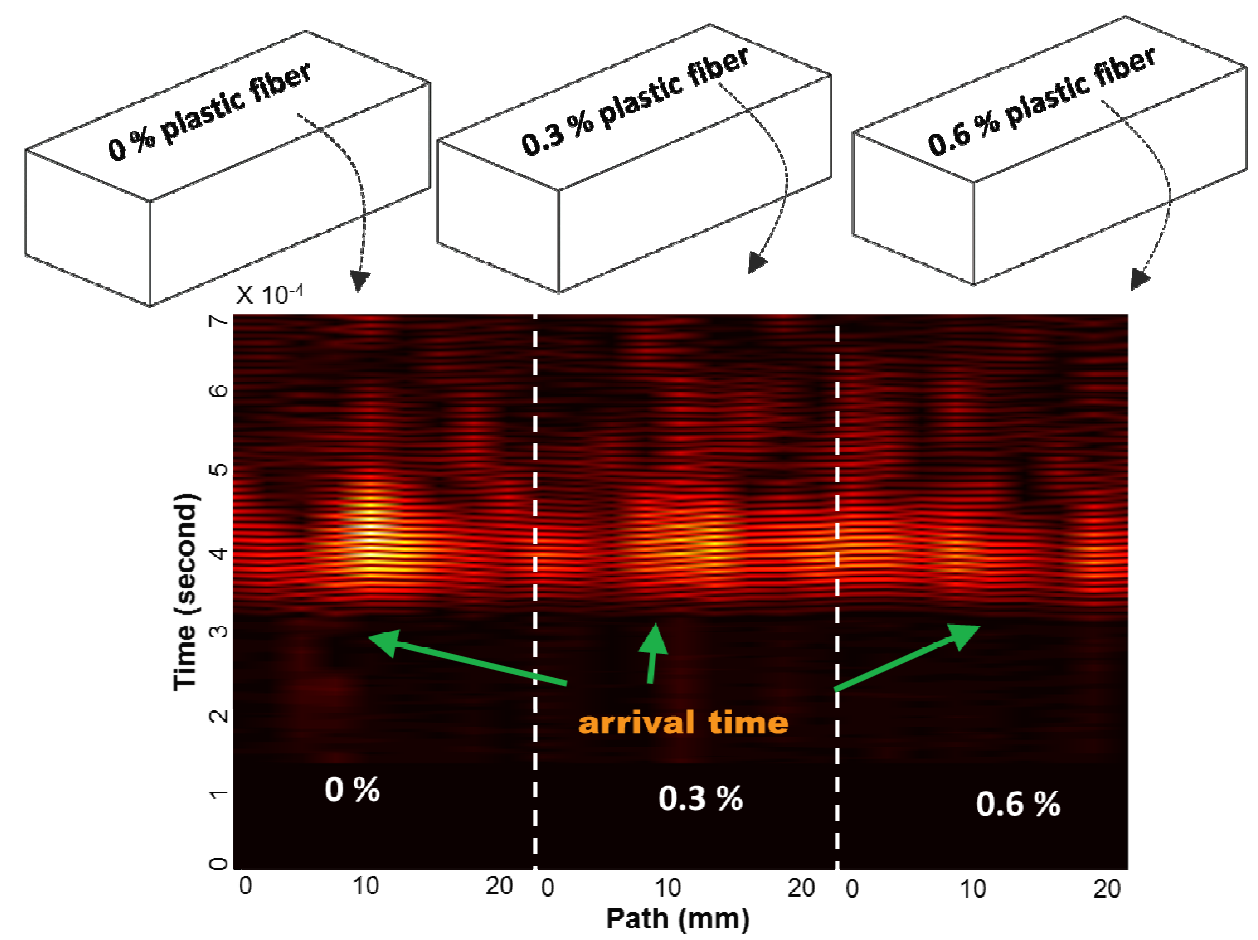

Figure 11. Fully contactless surface wave data with three different fiber contents $(0,0.3$ and $0.6 \%$ fiber content); multiple ultrasonic signals are stacked in the transverse direction across concrete samples. Surface wave arrival front indicated by arrows.

The signal energy results from a single path were determined; the results are shown in Figure 12. The energy content of the wave pulse in the signal, SE, is approximated by squaring the amplitude of the spatially averaged time signal and integrating the waveform between certain time points $\mathrm{t} 0$ and $\mathrm{t} 1$

$$
S_{E}=\int_{t 0}^{t 1}\left|v_{i}(t)\right|^{2}
$$

where $\mathrm{i}$ indicates path and $v$ is received time signal. In this work, $\mathrm{t}_{0}$ was set to the initial arrival time of the signal, and $t_{1}$ was defined by the arrival of the $5^{\text {th }}$ peak of the raw unprocessed signal. We found 
that energy results when $t_{1}$ was varied between the $3 r d$ to 8 th positive peak in the cyclic time signal provided consistent test to test results, while inconsistent results were obtained outside of that time range. Thus we selected in the middle of that time range (5th peak) to establish a consistent and transparent signal processing approach that is applied throughout. The arrival of the $5^{\text {th }}$ peak in the signal was determined using the AIC criterion. These test results show that surface wave amplitude attenuation is much more sensitive to the presence of the simulated damage than wave velocity. The energy attenuation shows a clear increasing trend with increasing fiber content (simulated cracking) as shown in Figure 11. These images give clear distinction of different fiber contents. The results suggest that the ultrasonic wave energy attenuation is a suitable basis by which to characterize microcracking levels in concrete.

The primary source of ultrasonic attenuation in the specimens is related to the number of fibers, whose interfaces scatter and attenuate the ultrasonic waves. The interference of the direct wave and multiply scattered waves results in a complex interference pattern that is apparent in the recorded time signals revealed over the whole time duration. It is noted that there is little distinction among the signals from the different samples at the early coherent portion of the signal pulse, thus conventional group velocity or initial amplitude cannot clearly distinguish among these different materials. The difference between the signals, as well as the effects of multiple scattering compounds themselves, become much more pronounced as the signal duration increases. It should be noted that contact sensor results, which are not reported in this paper, cannot distinguish among the fiber contents because of the variable nature of the coupling condition. Thus air-coupled ultrasonic testing has potential to characterize distributed damaged in the near-surface region in concrete. 

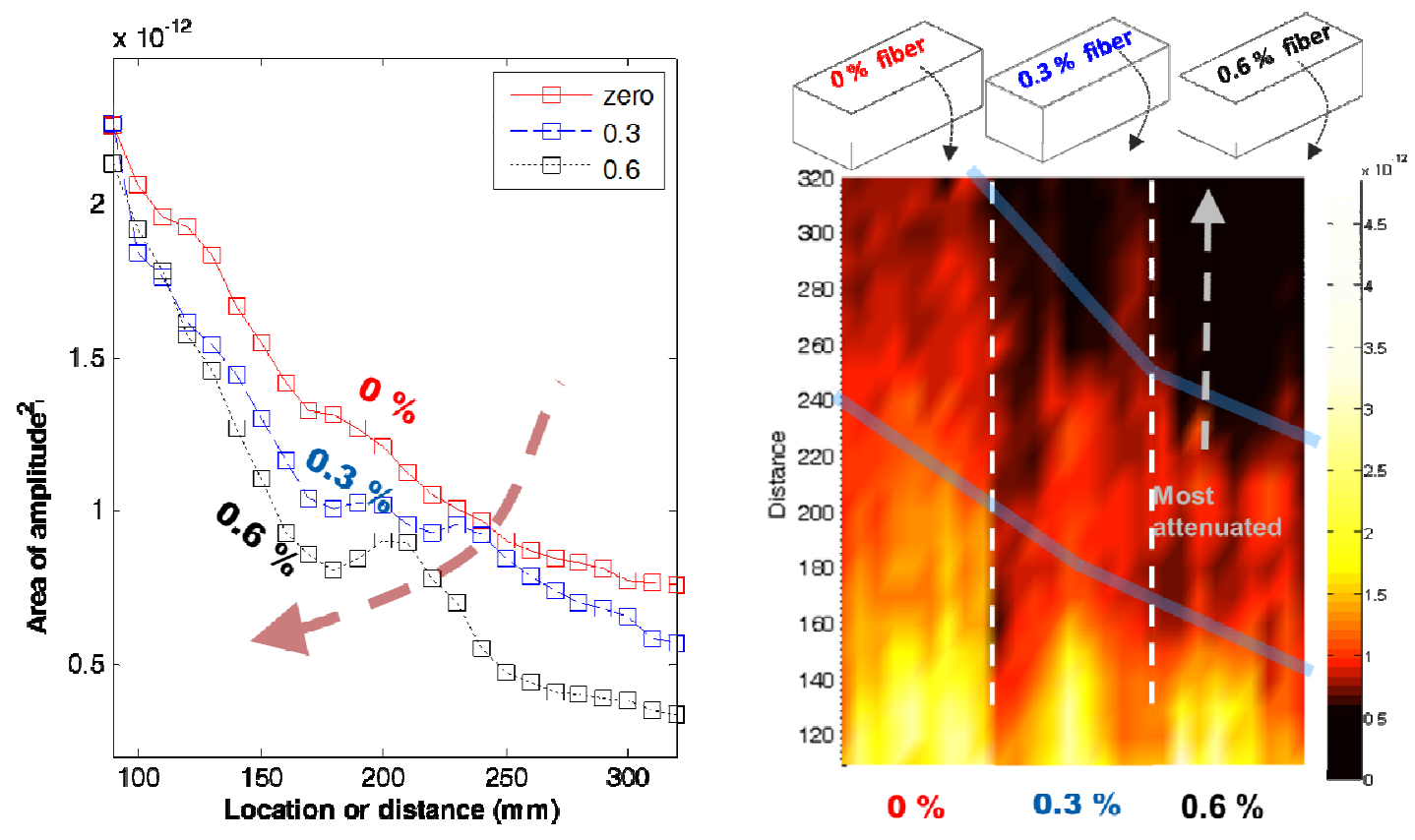

Figure 12. Energy attenuation of single line (left), and stacked color mapped imaged of multiple paths (right) for concrete samples with varying amounts of simulated damage.

\section{Conclusions}

We demonstrate that air-coupled transducers can launch and receive ultrasonic surface waves in concrete without direct contact, and further that the received signals have sufficiently high quality with low noise levels. The received surface wave characteristics, for example wave velocity and energy attenuation, are affected by the properties of the concrete with which the surface wave interacts. The resulting signal data can be analyzed and related to the condition of the concrete to detect and characterize embedded damage or flaws within the near-surface region. The technique is completely contactless (air-coupled sending and receiving) so the hardware is suitable for implementation in automated structural inspection systems, for example in automatic inspection using robots, providing quantifiable cost and time effort benefits.

The following conclusions about the specific testing system employed here are drawn, based on the results presented in this paper:

- Control of testing configuration parameters, such as sensor height and sender incident angle, is needed to generate high quality surface wave signals.

- The contactless sending (capacitive membrane) and receiving (MEMS) transducers provide 
consistent coupling conditions, allowing wave attenuation measurements to be carried out with improved sensitivity and confidence. The signal consistency of the air-coupled transducers is superior to that obtained with conventional contact sensors (accelerometers).

- The technique shows potential for efficient damage location and characterization within concrete elements, either through velocity or attenuation (wave energy) measurements. In particular, the surface wave signal data can distinguish RSD damage in concrete rail ties and detect varying amounts of simulated distributed microcracking damage in concrete blocks. The attenuation of the surface wave signal amplitude is much more sensitive to the presence of distributed damage than the surface wave velocity.

- Air-coupled sensors can provide suitable signals, even when applied to varying material surface roughness conditions.

\section{Acknowledgments}

The work reported in this paper was carried out under support from the National Science Foundation, through grant CMS1300546, and the American Association of Railroads through the Tech Scanning program.

\section{Cited References}

[1] N. Gucunski, F. Moon, ANDERS: future of concrete bridge deck evaluation and rehabilitation, in: H.F. Wu (Ed.), Proc. SPIE, 2011: pp. 798321-798321-9. doi:10.1117/12.881142.

[2] S. Iyer, S.K. Sinha, M.K. Pedrick, B.R. Tittmann, Evaluation of ultrasonic inspection and imaging systems for concrete pipes, Autom. Constr. 22 (2012) 149-164. doi:10.1016/j.autcon.2011.06.013.

[3] W. Wilson, V. Christopher, Aurora automated railroad tie condition assessment system: The quest for accuracy, in: AREMA 2009 Annu. Conf. Expo., 2009.

[4] ACI Committee 349, Evaluation of Existing Nuclear Safety-Related Concrete Structures, Rep. ACI 349.3R-02, Am. Concr. Institute, Farmington Hills, MI. (2002). 
[5] J.C. Zeman, Hydraulic mechanisms of concrete-tie rail seat deterioration, Ph.D. Dissertation, University of Illinois at Urbana-Champaign, 2010.

[6] ACI Committee 228, Nondestructive Test Methods for Evaluation of Concrete in Structures, Rep. ACI 228.2R-13, Am. Concr. Institute, Farmingt. Hills, MI. (2013).

[7] D.E. Chimenti, Review of air-coupled ultrasonic materials characterization, Ultrasonics. (2014). doi:10.1016/j.ultras.2014.02.006.

[8] P. Purnell, T.H. Gan, D.A. Hutchins, J.R. Berriman, Noncontact ultrasonic diagnostics in concrete: A preliminary investigation, Cem. Concr. Res. 34 (2004) 1185-1188. doi:10.1016/j.cemconres.2003.12.012.

[9] J.R. Berriman, P. Purnell, D.A. Hutchins, a Neild, Humidity and aggregate content correction factors for air-coupled ultrasonic evaluation of concrete., Ultrasonics. 43 (2005) 211-7. doi:10.1016/j.ultras.2004.07.003.

[10] J.R. Berriman, D. a Hutchins, A. Neild, T.H. Gan, P. Purnell, The application of timefrequency analysis to the air-coupled ultrasonic testing of concrete., IEEE Trans. Ultrason. Ferroelectr. Freq. Control. 53 (2006) 768-76.

[11] G.P. Cetrangolo, J.S. Popovics, Inspection of Concrete Using Air-Coupled Ultrasonic Pulse Velocity, ACI Mater. J. 107 (2010) 155-163.

[12] F. Soltani, M. Goueygou, Z. Lafhaj, B. Piwakowski, Relationship between ultrasonic Rayleigh wave propagation and capillary porosity in cement paste with variable water content, NDT\&E Int. 54 (2013) 75-83. doi:10.1016/j.ndteint.2012.12.003.

[13] M. Goueygou, Z. Lafhaj, F. Soltani, Assessment of porosity of mortar using ultrasonic Rayleigh waves, NDT\&E Int. 42 (2009) 353-360. doi:10.1016/j.ndteint.2009.01.002.

[14] O. Abraham, B. Piwakowski, G. Villain, O. Durand, Non-contact, automated surface wave measurements for the mechanical characterisation of concrete, Constr. Build. Mater. 37 (2012) 904-915. doi:10.1016/j.conbuildmat.2012.03.015.

[15] B. Piwakowski, M. Kaczmarek, P. Safinowski, Automated non destructive testing by noncontact surface waves, in: AIP Conf. Proc, Gdarisk, Poland, 2012: pp. 255-258. doi:10.1063/1.3703183. 
[16] V. Garnier, B. Piwakowski, O. Abraham, G. Villain, C. Payan, J.-F. Chaix, Acoustic techniques for concrete evaluation: Improvements, comparisons and consistency, Constr. Build. Mater. 43 (2013) 598-613. doi:10.1016/j.conbuildmat.2013.01.035.

[17] C.-W. In, J.-Y. Kim, K.E. Kurtis, L.J. Jacobs, Characterization of ultrasonic Rayleigh surface waves in asphaltic concrete, NDT\&E Int. 42 (2009) 610-617. doi:10.1016/j.ndteint.2009.04.007.

[18] B.L.J. Jacobs, J.O. Owino, Effect of aggregate size on attenuation of Rayleigh surface waves in cement-based materials, J. Eng. Mech. 126 (2000) 1124-1130.

[19] G. Hevin, O. Abraham, H.A. Pedersen, M. Campillo, Characterisation of surface cracks with Rayleigh waves: a numerical model, NDT\&E Int. 31 (1998) 289-297.

[20] K.F. Graff, Wave Motion in Elastic Solids, Dover Publications, New York, 1991.

[21] J. Zhu, J.S. Popovics, F. Schubert, Leaky Rayleigh and Scholte waves at the fluid-solid interface subjected to transient point loading, J. Acoust. Soc. Am. 116 (2004) 2101. doi:10.1121/1.1791718.

[22] G.D. Maslin, A simple ultrasonic ranging system, J. Acoust. Soc. Am. 73 (1983) S58. doi:10.1121/1.2020465.

[23] H. Carr, C. Wykes, Diagnostic measurements in capacitive transducers, Ultrason. 31 (1993) $13-20$.

[24] B. Siciliano, O. Khatib, Part1 : Springer Handbook of Robotics, Springer Berlin Heidelberg, Berlin, Heidelberg, 2008. doi:10.1007/978-3-540-30301-5.

[25] L.C. Lynnworth, Ultrasonic impedance matching from solids to gases, IEEE Trans. Son. Ultrason. SU-12 (1965) 37-48.

[26] W. Manthey, N. Kroemer, V. Magori, Ultrasonic transducers and transducer arrays for applications in air, Meas. Sci. Technol. 3 (1992) 249-261.

[27] J.A. Walraven, Introduction to applications and industries for microelectromechanical systems (MEMS), Int. Test Conf. 2003. Proceedings. ITC 2003. 1 (2003). doi:10.1109/TEST.2003.1270896. 
[28] J. Zhu, J.S. Popovics, Imaging Concrete Structures Using Air-Coupled Impact-Echo, J. Eng. Mech. 133 (2007) 628-640.

[29] T. Oh, J.S. Popovics, S. Ham, S.W. Shin, Improved Interpretation of Vibration Responses from Concrete Delamination Defects Using Air-Coupled Impact Resonance Tests, J. Eng. Mech. 139 (2013) 315-324. doi:10.1061/(ASCE)EM.1943-7889.0000491.

[30] B.J. Lewis, B. Moss, MEMS Microphones, the Future for Hearing Aids, Analog Dialogue. 47 (2013) 3-5.

[31] S. Mindess, J.F. Young, D. Darwin, Concrete (2nd Edition), Prentice Hall, 2002.

[32] J.C. Zeman, J.R. Edwards, C.P.L. Barkan, D.A. Lange, Failure mode and effect analysis of concrete ties in North America, in: Proc. 9th Int. Heavy Haul Conf., Shanghai, China, 2009: pp. $220-277$.

[33] D.W. Schindel, D.A. Hutchins, M. Sayer, The design and characterization of micromachined air-coupled capacitance transducers, IEEE Trans. Ultrason. Ferroelectr. Freq. Control. 42 (1995) 42-50. doi:10.1109/58.368314.

[34] J.A. Jensen, S.I. Nikolov, K.L. Gammelmark, M.H. Pedersen, Synthetic aperture ultrasound imaging., Ultrasonics. 44 Suppl 1 (2006) e5-15. doi:10.1016/j.ultras.2006.07.017.

[35] D.G. Aggelis, T. Shiotani, Effect of Inhomogeneity Parameters on Wave Propagation in Cementitious Material, ACI Mater. J. (2009) 187-193. 